\title{
Spatial and spectral characteristics of spontaneous emission from semiconductor quantum wells in microscopic cylindrical cavities
}

\author{
Igor Vurgaftman ${ }^{\text {a) }}$ \\ Code 5613, Naval Research Laboratory, Washington, DC 20375 \\ Jasprit Singh \\ Department of Electrical Engineering and Computer Science, The University of Michigan, Ann Arbor, \\ Michigan 48109-2122
}

(Received 29 June 1995; accepted for publication 18 October 1995)

\begin{abstract}
The spontaneous emission rate from an $\operatorname{In}_{0.2} \mathrm{Ga}_{0.8} \mathrm{As}$ quantum well in a cylindrical dielectric cavity with submicron radii and without mirrors in the emission plane is calculated based on a rigorous description of the cavity modes and their interaction with the $2 \mathrm{D}$ electronic system. The rate is suppressed for radii much less than the peak optical wavelength $\left(r_{\text {cav }} \ll \lambda / n\right)$, enhanced by up to a factor of $\approx 8$ for $r_{\text {cav }} \approx \lambda / n$, and remains similar to its value in the absence of a cavity for $r_{\text {cav }}$ $\gg \lambda / n$. The emitted light produces a highly collimated vertical beam in the intermediate region with the majority of photons emitted into the guided cavity modes, and its spatial distribution broadens dramatically in the smallest structures. The global spectral width of spontaneous emission in cavities that can presently be fabricated is largely unaffected by the introduction of lateral dielectric boundaries. (C) 1995 American Institute of Physics.
\end{abstract}

Vertical light emitter arrays are very attractive for massively parallel optical interconnects. ${ }^{1}$ In recent years, considerable progress has been made in the development of vertical-cavity surface-emitting lasers (VCSELs) with distributed Bragg reflectors (DBRs) providing optical feedback. ${ }^{2}$ Additional excitement has been sparked by the suggestion that microcavity effects occurring in optical cavities with dimensions of the order of the wavelength of the light might be used to improve the performance of VCSELs. ${ }^{3,4}$ The greatest control over spontaneous emission can be exercised in an index-guided VCSEL with tight lateral confinement. ${ }^{4,5}$ Unfortunately, the presence of DBR mirrors makes the fabrication of the device and efficient current injection into it quite difficult. The effects of surface recombination may be invoked to account for the fact that so far, contrary to theoretical predictions, the threshold current density in VCSELs increases when the cavity radius is reduced. ${ }^{6}$ Apparently, optimization of the fabrication process can aid in ameliorating the state of affairs; nevertheless, the question of whether microcavity effects in surface light-emitting structures simpler than the present-day VCSELs can be utilized with beneficial influence on the device performance remains.

The fabrication and current injection problems can be partially resolved by removing the DBR mirrors [see Fig. 1(a)]. Lasing action has been demonstrated in microcylinders with radii much greater than the emission wavelength and attributed to the high $Q$ of the whispering-gallery modes. ${ }^{7,8}$ Microcylinders with radii comparable to the optical wavelength lack high- $Q$ modes so that stimulated emission in them is not sufficient to commence laser oscillation. Nonetheless, they are of interest since modified spontaneous emission determines the properties of the light output in the LED regime of operation.

${ }^{a)}$ Electronic mail: vurgaftman@tantalus.nrl.navy.mil
In many applications, LEDs are more likely to be employed than laser diodes, primarily owing to the much poorer reliability of laser diodes. ${ }^{1}$ This is expected to be true for massively parallel interconnection of electronic chips, where at issue is the directionality of the emitted light, not its coherence. In this letter, we consider whether the spontaneous emission rate and its spatial and spectral distributions can be greatly affected by the introduction of lateral dielectric boundaries into a mirrorless cavity.

To answer these questions, it is necessary, first of all, to obtain an accurate description of the modes in the dielectric cavity, which may be classified into (1) guided modes oscillating inside the cavity, decaying outside, and carrying electromagnetic energy along the cylinder axis, and (2) radiation modes oscillating both inside and outside and carrying energy in arbitrary off-axis directions. As in the optical fiber problem, ${ }^{9}$ the guided mode profiles are Bessel functions inside the cavity and decay asymptotically as Hankel functions of the first kind of the imaginary argument beyond. Assuming that the cylinder length is much greater than its radius and that the variation of the refractive index along the axis is smooth as compared to the radial variation, the propagation constant $\beta$ takes on discrete values $m \pi / L$, where $m$ is a positive integer, and $L$ is the length of the cylinder, taken in the following to be $20 r_{\text {cav }}$. The primary effect of adopting a finite length of the cylinder is that at very small cavity radii, the $\mathrm{HE}_{11}$ mode of the cavity (which does not have a cutoff if the cavity length is infinite) is not supported since the corresponding wave number is smaller than $\pi / L$. The guided modes fall into the range of frequencies $\beta c / n_{1}<\omega$ $<\beta c / n_{2}$, where $n_{1}$ is the refractive index of the core, and $n_{2}$, of the cladding. The modes are found numerically by incrementing the frequency in the allowed range and looking for the solutions of the eigenvalue equation. Once a mode is found, its amplitude is determined by integrating the energy density and setting the modal energy to its zero-point value. 

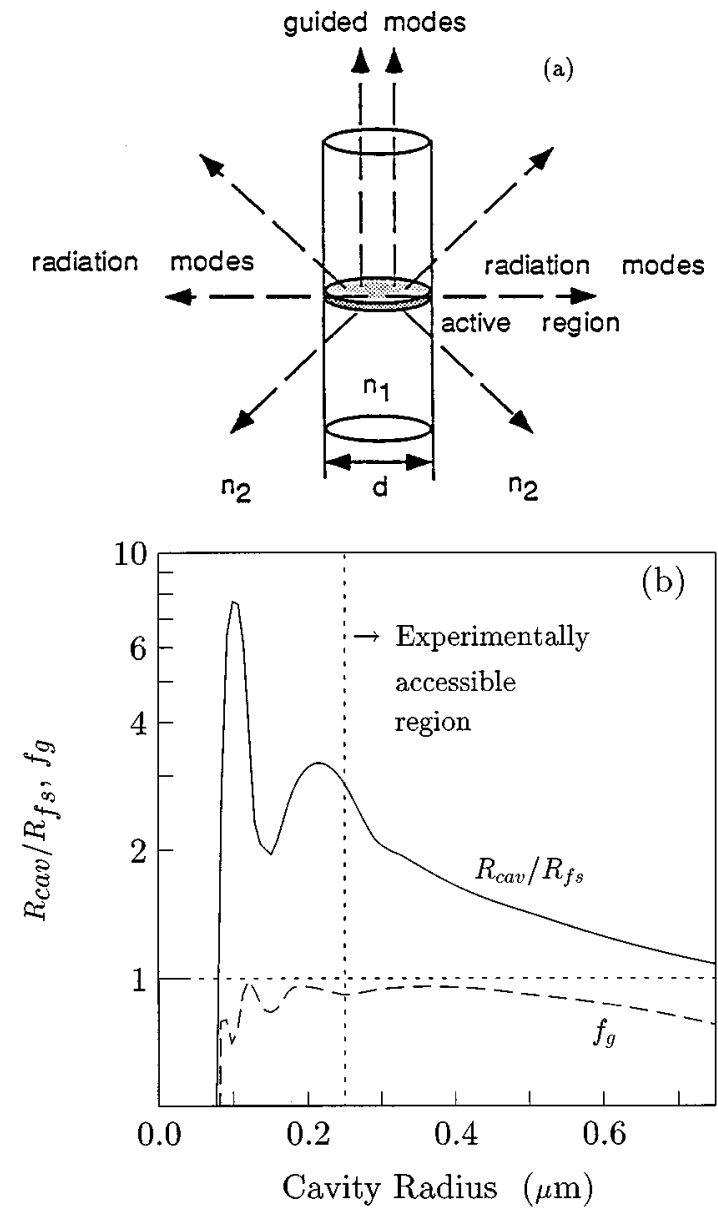

FIG. 1. (a) A schematic of the considered microcavity LED that has no DBR mirrors in the emission plane and derives optical confinement from the GaAs-air $\left(n_{1} \approx 3.6, n_{2}=1.0\right)$ boundary. (b) The ratio of the spontaneous emission rate in the microcylinder to that in the absence of a cavity for the same active area (solid line), and the fraction of photons emitted into the guided optical modes of the cavity (dashed line) as a function of the cavity radius for a carrier density of $2 \times 10^{18} \mathrm{~cm}^{-3}$.

In contrast to guided modes, radiation optical modes form a continuum and satisfy the $\omega>\beta c / n_{2}$ condition. The modal profiles are Bessel functions inside the cavity and linear combinations of Bessel and Neumann functions outside. It is convenient to treat the radiation modes as quasicontinuous modes of a very large conductor cavity by analogy with the standard quantum-mechanical normalization procedure. With these assumptions, the eigenvalue equations for the radiation modes can be derived and solved numerically in a manner similar to that previously outlined for the guided modes.

The expression for the spontaneous emission rate in the cavity can be obtained by substituting the mode amplitudes determined by the cavity mode calculation for the free-space value $\mathscr{C}=i \sqrt{\hbar \omega_{k} / 2 \epsilon V} \exp (i \mathbf{k} \cdot \mathbf{r})$ in the usual expression originating from the Fermi golden rule:

$$
\begin{aligned}
\frac{1}{\tau_{\mathrm{sp}}}= & \frac{2 \pi}{\hbar} \sum_{\text {modes }} \frac{e^{2}}{m_{0}^{2} \omega_{\text {mode }}^{2} \boldsymbol{\kappa}, s}\left|\left\langle v\left|\mathbf{P} \mathscr{C}_{\text {mode }}(\mathbf{r})\right| c\right\rangle \cdot \boldsymbol{\varepsilon}_{\text {mode }}\right|^{2} \\
& \times \delta\left(E_{c}\left(\boldsymbol{\kappa}_{c}\right)-E_{v}\left(\boldsymbol{\kappa}_{v}\right)-\hbar \omega_{\text {mode }}\right)
\end{aligned}
$$

$$
f_{e}\left[E_{c}\left(\boldsymbol{\kappa}_{c}\right)\right] f_{h}\left[E_{v}\left(\boldsymbol{\kappa}_{v}\right)\right],
$$

where $\boldsymbol{\varepsilon}$ is the unit polarization vector, $f_{e}$ and $f_{h}$ are the electron and hole occupation function, respectively, $\boldsymbol{\kappa}$ is the $2 \mathrm{D}$ electron wave vector, $E_{c}$ and $E_{v}$ are the energies in the conduction and valence band, respectively, and $s$ is the spin index. Making the assumptions of a single subband in the conduction and valence bands with an isotropic effective mass, parabolic dispersion and zone center Bloch functions $|s\rangle$ in the conduction band and $1 / \sqrt{2}\left(\left|p_{x}\right\rangle \uparrow+i\left|p_{y}\right\rangle \uparrow\right)$ or $i / \sqrt{2}\left(\left|p_{x}\right\rangle \downarrow-i\left|p_{y}\right\rangle \downarrow\right)$, depending on the spin, in the valence band, and that both bands are spin-degenerate, the band edge states of a narrow compressively strained InGaAs quantum well can be represented to the lowest order. Approximating the integral over the envelope wave functions as unity, and assuming that the magnitude of the radial electron wave vector is much greater than the radial photon wave vector and that the electron azimuthal index is much greater than the optical one, the following expression for the matrix element can be derived: ${ }^{10}$

$$
\left|M_{c v}\right|^{2}=\frac{\pi^{2}}{a^{2}}\left[\left|\int_{0}^{a} \mathscr{A}_{r} d r\right|^{2}+\left|\int_{0}^{a} \mathscr{C}_{\phi} d r\right|^{2}\right] P_{c v}^{2},
$$

where $P_{c v}=\left\langle p_{x}\left|P_{x}\right| s\right\rangle=\left\langle p_{y}\left|P_{y}\right| s\right\rangle$. The spontaneous emission rate in terms of the joint electronic density of states is

$$
\begin{aligned}
\frac{1}{\tau_{\mathrm{sp}}}= & \frac{2 \pi}{\hbar} \sum_{\text {modes }} \frac{e^{2}}{m^{2} \omega_{\text {mode }}^{2}}\left|M_{c v}^{\text {mode }}\right|^{2} V\left(\frac{\rho(\kappa)}{d E_{\mathrm{eh}} / d \kappa}\right)_{E_{\mathrm{eh}}=\hbar \omega_{\text {mode }}} \\
& \times f_{e}\left(E_{c}\right) f_{h}\left(E_{v}\right)
\end{aligned}
$$

where $V$ is the volume of the active region. The quasi-Fermi levels in the conduction and valence bands are adjusted so that the electron and hole densities in the intrinsic active region are equal. The spontaneous emission rate in the absence of a cavity can be found by converting the sum over the photon states into a frequency integral using the 3D density of states multiplied by factors of 2 for degenerate linear polarizations of a plane wave mode and one third for the average over zone center Bloch functions. ${ }^{11}$ The spatial profile of spontaneous emission in the cavity plane is obtained as the differential energy per unit area in each mode weighted by the fraction of all photons emitted into the mode, approximated by the emission fraction. The full formalism will be presented separately. ${ }^{10}$

The calculations were performed for a GaAs cavity surrounded laterally by air. The results for the ratio of the spontaneous emission rate in the cavity to that without a cavity and with the same active area $\left(R_{\mathrm{cav}} / R_{\mathrm{fs}}\right)$ as a function of the radius is shown in Fig. 1(b) for an injected carrier density of $n=2 \times 10^{18} \mathrm{~cm}^{-3}$. Unfortunately, the mode calculation becomes impractically cumbersome and time consuming and the formalism less exact for larger radii. Nevertheless, it is apparent from Fig. 1(b) that the spontaneous emission rate in this case tends to the free-space rate. When the cavity radius becomes comparable to the peak wavelength of the light divided by the refractive index of the core $\left(\lambda_{r}\right)$, a considerable enhancement in $R_{\text {cav }} / R_{\mathrm{fs}}$ and the fraction of photons emitted into the guided modes of the cavity $f_{g}$ is observed. While in 


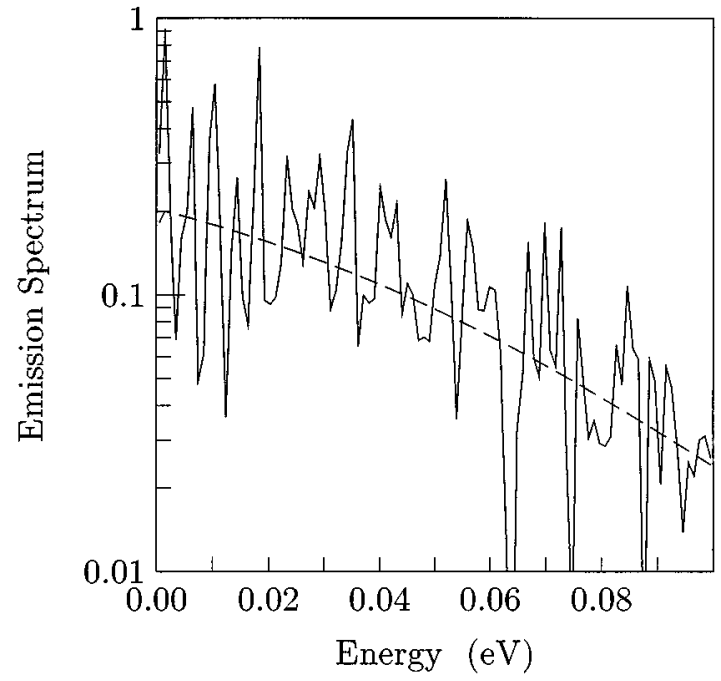

FIG. 2. The spectral distribution of spontaneous emission in a $0.5 \mu \mathrm{m} \mathrm{mi-}$ crocylinder (solid line) and in the absence of a cavity (dashed line). The photon energy is measured from the band gap energy of the quantum well comprising the active region.

a macroscopic cavity, emission occurs into a continuum of states, the spectral extent of which is much greater than that of the electron-hole pairs in the active region, in a microcavity, a single, spectrally distinct, mode can receive a large fraction of spontaneous emission. Therefore, the changes in the position of the discrete modes with respect to the band gap of the InGaAs quantum well, are directly reflected in the oscillations of $R_{\text {cav }} / R_{\mathrm{fs}}$ and $f_{g}$ as a function of the cavity radius. However, the dimensions necessary to isolate a few modes near the band gap energy are impractically small for the current fabrication technology (a radius of $\approx 0.1 \mu \mathrm{m}$ ). In such cavities, a single mode influences the degree of enhancement in the spontaneous emission rate. At higher carrier densities, the Fermi level does not penetrate as far into the bands, and the enhancement over free space decreases as the emission rate grows. In wider cavities, $R_{\text {cav }} / R_{\mathrm{fs}}$ and $f_{g}$ are not sensitive to the carrier density. As the unbroadened spectral profile of spontaneous emission for a cavity with a $0.5 \mu \mathrm{m}$ radius shown in Fig. 2 indicates, the large number of modes in the emission bandwidth causes a global spectral width similar to that in the absence of the cavity to be maintained for cavities with radii greater than $0.25 \mu \mathrm{m}$. The unbroadened spectrum is considered here for simplicity since the exact broadening mechanism and extent depend on the details of the cavity losses and carrier scattering. The peak at the band gap energy is due to the assumption of a single isotropic parabolic band. The results for the emission bandwidth contrast with those for a cavity with DBR mirrors, which provide the wavelength selectivity necessary to reduce the spectral extent of spontaneous emission.

The spatial profile of spontaneous emission is shown in Fig. 3 for several cavities. For cavities with $r_{\text {cav }}<\lambda_{r} / 4$, the output beam diverges since almost all of the power is concentrated in the radiation modes. On the other hand, for cavities with the radius approaching the optical wavelength, the output beam is vertically collimated with an intensity peak

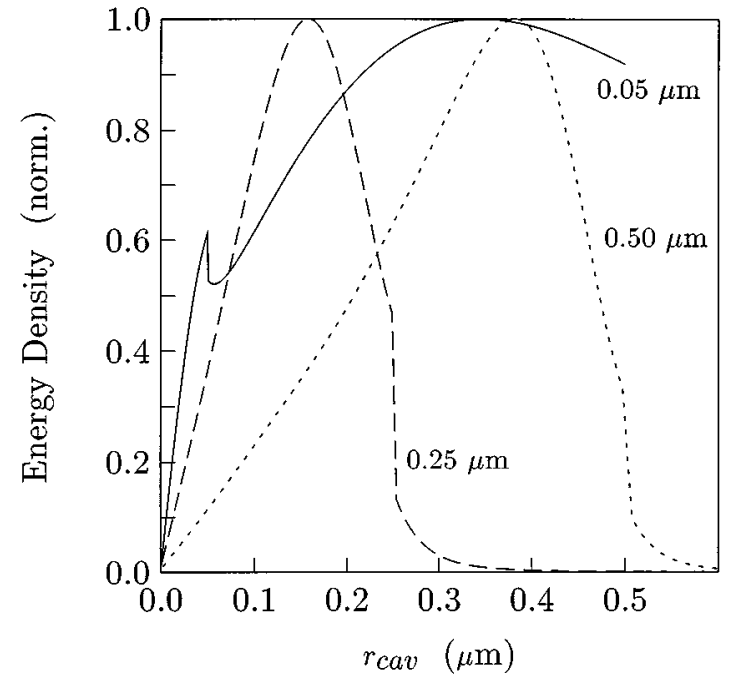

FIG. 3. The normalized spatial distribution of spontaneously emitted radiation approximated in terms of the energy density as a function of the distance from the origin for several cavity radii.

close to the origin. The peak shifts away from the origin for larger cavities returning to a broad spatial profile in the limit of vanishing lateral confinement. In optical interconnect applications, the photon flux per unit solid angle in a given direction must be maximized. ${ }^{1}$ Since here DBR mirrors are not required to achieve this effect, the number of growth steps can be greatly reduced. This makes the $r_{\text {cav }} \approx \lambda_{r}$ structures candidates for highly efficient and reasonably practical sources for optical interconnections requiring surface emitters.

Work at the University of Michigan was supported by the Advanced Research Projects Agency, MDA 972-94-10004. The content of this letter does not necessarily reflect the position of the Government. Part of this work was performed while one of the authors (I.V.) held a National Research Council-NRL Research Associateship and was supported by the Office of Naval Research.

${ }^{1}$ E. F. Schubert, N. E. J. Hunt, M. Micovic, R. J. Malic, S. L. Sivco, A. Y. Cho, and G. J. Zydzik, Science 265, 943 (1994).

${ }^{2}$ K. Iga, F. Koyama, and S. Kinoshita, J. Vac. Sci. Technol. A 7, 842 (1989).

${ }^{3}$ G. Bjork, H. Heitmann, and Y. Yamamoto, Phys. Rev. A 47, 4451 (1993).

${ }^{4}$ T. Baba, T. Hamano, F. Koyama, and K. Iga, IEEE J. Quantum Electron. QE-27, 1347 (1991).

${ }^{5}$ I. Vurgaftman and J. Singh, IEEE J. Quantum Electron. QE-31, 852 (1995).

${ }^{6}$ K. Iga, F. Koyama, S. Kinoshita, IEEE J. Quantum Electron. QE-24, 1845 (1988).

${ }^{7}$ A. F. J. Levi, R. E. Slusher, S. L. McCall, S. J. Pearton, and W. S. Hobson, Appl. Phys. Lett. 62, 2021 (1993).

${ }^{8}$ A. F. J. Levi, R. E. Slusher, S. L. McCall, J. L. Glass, S. J. Pearton, and R. A. Logan, Appl. Phys. Lett. 62, 561 (1993).

${ }^{9}$ D. Marcuse, Light Transmission Optics (Van Nostrand Reinhold, New York, 1982).

${ }^{10}$ I. Vurgaftman and J. Singh (unpublished).

${ }^{11} \mathrm{~J}$. Singh, Physics of Semiconductors and Their Heterostructures (McGraw-Hill, New York, 1993). 